scheint die Auffassung von Campbell und Johnson zu sein, denn sie erklären fast alles, was absolut nicht auf die Marchantiales stimmt, als Anpassung an das "peculiar habitat" resp. an die subaquatische Lebensweise. Daß letzteres unmöglich richtig sein kann, habe ich schon früher nachgewiesen, auch zeigen wirklich aquatische, primitive Formen der Marchantiales, wie z. B. Riccia fluitans und Ricciocarpus natans ein wohl entwickeltes Luftkammergewebe resp. auch sehr mächtige Ventralschuppen. Eine so hochgradige Anpassung, welche gerade die fundamentalsten Merkmale zum gänzlichen Verschwinden bringt, wäre überhaupt nur denkbar bei einer höchstgradig abgeleiteten Form, ist aber völlig ausgeschlossen bei einer primitiven Form.

\title{
Die ungarisch-österreichische Flora des Carl Clusius vom Jahre 1583.
}

\author{
Von Dr. Hermann Christ (Basel).
}

(Schluß. ${ }^{1}$ )

\section{Orientalische Einführungen.}

Außer seiner einheimischen Flora verbreitet sich nun Clusius, im Anschluß an deren Genera, über eine stattliche Menge eingeführter Pflanzen, und seine Ausführungen sind um so wichtiger, als gerade damals und nicht zum geringsten Teil durch die Tätigkeit des Clusius, eine wahre Einwanderung so vieler Blumen und Bäume aus dem Orient über Konstantinopel stattfand, welche jetzt noch den eisernen Bestand unserer Gärten bilden. Seit den Kreuzzügen sind jedenfalls nie so viel exotische Gewächse lebend nach Wien und über Wien nach Belgien und weiterhin eingeführt worden als gerade damals, und es ist das hohe Verdienst des Clusius, uns so genaue Nachricht über diese Einführungen gegeben zu haben. Die Kapitel namentlich der Tulpen, Lilien, Anemonen und Iris hat er geradezu monographisch behandelt. Da unter einem Kaiser, wie Rudolf JI., der sich um das Reich sehr wenig, um so mehr aber um seine Liebhabereien: Astronomie (Kepler und Tycho de Braho waren seine Hofastronomen) und Sammlungen aller Art, auch um die Pflanzenwelt bekümmerte, sich die Gärten von Wien bereicherten, ist weniger merkwürdig, als daß in Konstantinopel unter Sultanen wie dem schrecklichen Soliman II, und während der beständig tobenden Kriege gegen Ungarn und Österreich die Gartenkunst und die Kultur einer Masse asiatischer Blumen, besonders Zwiebelpflanzen und Anemonen, blühte. Günstig war es nun, daß zu Clusius' Zeit das Personal der kaiserlichen Gesandtschaft in Konstantinopel zu großem Teil aus ihm befreundeten Niederländern bestand, die teils ihm direkt, teils den Hofherren in Wien immer neue Sendungen von Zwiebeln und lebenden Gewächsen machten. Über diese Einführungen hat nun Clusius in der pannonischen Flora genau Buch geführt. Diese Gesandtschaftsmitglieder waren namentlich David Ungnad v. Sonneck, Philibert de Bruxelles, Stephan จ. Hansen, Carl Rym, de Eeckebecke und andere. Die Gartenbesitzer

1) Vgl. Nr. 3, S. 131. 
Wiens, welche die Gaben dieser Herren empfingen, waren Wolfgang Christoph v. Entzestorf, Hofmarschall Paul v. Trautzon, Frau v. Heijsenstein u. a. Dies bekundet einen regen Verkehr und einen aufgeschlossenen Sinn für die Wissenschaft, wie man ihn in jener trüben Zeit, wo der Kammerdiener Lang und der Kardinal Klesl das Reich regierten, nicht erwarten durfte.

Von diesen Erwerbungen der europäischen Gartenflora aus der Türkei heben wir folgende hervor:

Den Laurocerasus S. 2 (der Name rührt von Bellonius her) führt dieser Orientreisende des 16. Jabrhundert von Trapezunt an, glaubt auch, ihn im Garten des Fürsten Doria in Genua gesehen zu haben, aber erst 1574 kamen trockene Früchte davon nach Wien, und 1576 sandte der kaiserliche Gesandte in Konstantinopel, David Ungnad, den Strauch lebend an Clusius, unter Angabe des türkischen Namens Trabison Kuzmosi-Dattel von Trapezunt. Im Jahre 1583 blühte dann ein Exemplar in einem Topfe bei Dr. Aicholtz, woron Clusius im Anhang S. 2 einen Zweig abbildet.

Die Castaneu equina (Aesculus) hat zwar schon der Amtsvorgänger des Clusius, Matthiolus, beschrieben, aber da Clusius den Baum lebend beobachtete, was bisher noch nie der Fall war, schildert er ibn ausführlich, namentlich auch die großse, mit einem fetten, klebrigen Saft bedeckte Knospe, an welcher Mücken und andere Insekten hängen bleiben. Die Blüte ist ihm noch unbekannt, dagegen erbielt er $1581 \mathrm{die}$ Frucht aus Konstantinopel. Den Alten ist nach Clusius der Baum unbekannt geblieben. Die 'Türken nennen ihn at ceestanesi $=$ Roßkastanien und heilen mit der Frucht dämpfige und hustende Pferde.

Auch die Platanus hat Clusius gleichzeitig mit dem Kirschlorbeer und der Roßkastanie aus der Türkei erhalten. Für die Beschreibung verweist er auf die älteren Autoren.

Nun die Tulpen.

Clusius teilt die Gartentulpen ein in zwei Genera:

1. Praecoces, 2. Serotinae, zwischen denen eine Mittelform: Dubiae auftritt.

In den Stirp. Hisp., Append. 510 (1576), sagt Clusius, die Praecoces und Serotinae seien nicht durch die Gestalt, sondern nur durch die Blütezeit verschieden. In den Stirp. Pannon. 145 behandelt er sie jedoch als Genera primaria, als besondere Spezies.

Erstere sind die kleinen, frühblühenden Formen, im allgemeinen wohl der T. praecox Ten. nicht unähnlich, wie sie noch wild im mediterranen Gebiete vorkommt. Die Serotinae sind spätblühend, viel größer, eine Elle bis drei Fuß boch, öfter ästig, Blüte länglich, Blatt fester, Kapsel dicker, Samen und Zwiebeln größer, im übrigen den Praecoces gleich. Offenbar bilden die serotinae das, was wir heute mehr oder weniger unter $T$. Gesneriana verstehen.

Innerbalb dieser zwei Gruppen bringt nun Clusius seine Formen nach Merkmalen der Farbe und Zeichnung der Sepalen unter. I. Praecoces. Von einfarbigen nennt und beschreibt er 1. gelbe, 2. rote, von denen einige die bei vielen wilden Tulpen gewöhnlichen gelben Nagelflecke mit schwarzem Zentrum haben, 3. weiße, 4. purpurne bis violette. 
Unter 5. kommt er dann auf die Variae, die gescheckte oder wechselnde Farben zeigen. Er ordnet sie nach den Hauptfarben a) gelb, in 8 verschiedenen Modifikationen, von denen er sagt, daß einige anfangs ganz gelb sind und erst am 4. oder 5. Tage rot gescheckt werden. b) Rot in ebenfalls 8 Formen. c) Weiß, wiederum mit 8 Abänderungen. d) Purpur in 9 Formen: Alle so genau beschrieben, daß man sie beinahe heute nachmalen könnte. Bekanntlich gibt es Stellen, wo solche Tulpen heute in verwirrender Farbenvarietät noch wild vorkommen: so bei Florenz, in der Maurienne ete. (vergl. Solms-Laubach, Weizen und Tulpen 1898). Es wäre der Mühe wert, diese mit den Formen des Clusius zu konfrontieren.

II. Serotinae. Hier ist die Versebiedenheit der Farben weniger groß: wesentlich nennt er nur zwei: gelbe und rote. Die Serotina rubro fält nach Clusius Beschreibung 164 mit der T. oculus Solis St. Am., so wie sie haute noch neben der kleineren, in allen Teilen breiteren. stumpferen T. praecox bei Florenz vorkommt, genau zusammen. Clusius beschreibt sehr genau die zweifarbige Zeichnung des Nagels der Sepala und fügt den Vulgärnamen Ochio di sole, hoe est solis oculus bei.

Dubiae. Diese schwanken zwischen beiden Hauptkategorien; von kleineren Formen zählt Clausius drei, von größeren eine auf.

Über seine Aussaatversuche meldet uns Clusius folgendes:

Sehr wenige der Sämlinge ergeben die Farbe der Mutterpflanze, sondern die meisten gehen, namentlich bei den Praecoces, in verschiedene Farben über, degenerieren auch oft ins gelbe. Namentlich ergeben die weißen ganz ausgezeichnete Verschiedenheit im Kolorit. "Samen derselben Kapsel, von mir selbst gesammelt und gleichzeitig der Erde übergeben, haben im nächsten Frühling einige Pflänzchen ergeben, die nach dem 5 . bis 10. Jahre (denn manche entwickeln sich rascher als andere) weiße, weißgescheckte, gelbe, gelbgescheckte, rote, rotgescheckte und purpurne und purpurgescheckte Tulpen ergaben." Schade, daß Clusius noch nicht :e exakte Methode Gregor Mendels anzuwenden wußte!

Bei den Serotinae pflegen die Sämlinge die Farbe der Mutter zu bewahren, höchstens daG, wenn sie überhaupt eine "Mutatio" erwerben, diese sich am Nagel der Sepalen oder den Staubfäden äußert. Klingt diese erworbene Mutatio in diesem Zusammenhang nicht wie eine Ahnung hentiger Theorien?

Im Anhang zur Pannonischen Flora teilt Clusius das Resultat neuer Aussaaten von 1575 und 1576 mit, deren Samen ihm Augerius v. Bousbeque aus Konstantinopel gesandt hatte. Die Verschiedenheit der Farben war eine erstaunliche, weitaus die Mehrzahl den Praecoces angehörig, aber doch auch einige, welche in bezug auf Blütezeit und Größe den Serotinae oder den Dubiae nahe standen, welche aber Clusius doch wegen der bunten Farben von den Praecoces nicht trennen will, da er die Serotinae für viel farbenbeständiger hält, indem ihm sowohl die roten als die gelben in der Aussaat wiederum Tulpen gleicher Farbe und nie bunte gaben; höchstens ergab etwa eine rote eine gelbe und umgekehrt, oder es zeigten sich bei der Aussaat von Serotinae und Dubiae abgeblaßte, trüb und grünlichgelbe bis weißliche oder dann trübe rote Formen. 
Schade, daf Clusius bei seinen Tulpenstudien fast ausschlieflich auf die Farbe geachtet und nicht anderweitige Unterschiede eben so konsequent verfolgt hat.

Von den Praecoces ist die rote und die weiße die fruchtbarste, von allen aber am meisten die rote Serotina, die schon im 2. Jahre, ehe sie zu blühen beginnt, Brutzwiebeln macht, während die Zwiebel tief in die Erde sich senkrecht hinabsenkt.

In der alten Zwiebelhülle bleibt dann etwas lebender Substanz, die in eine neue Zwiebel auswächst und so fort, so dab jemand, der sorgfältig zwei oder drei solcher Brutzwiebeln ausgräbt, indem er die Reste der alten Hüllen verfolgt, eine Kette zusammenhängender Zwiebeln nachweisen kann, die alle der ersten entstammen: Die Gartentulpen pflegen jung und vor der Blüte alle Jahre mehr in die Tiefe zu sinken, während die wilden Arten vor der Blüte nicht in die Tiefe, sondern seitwärts und schief an langen Fäden ihre Brut verbreiten. „Ich erinnere mich, aus einer in der Achsel des untersten Blattes entstandenen Brutzwiebel (Bild 167), dieselbe Farbe wie die Mutterpflanze erbalten zu haben; dasselbe ist bei allen Brutzwiebeln der Fall.“

Der Stengel pflegt mitten aus der Zwiebel hervorzukommen. Wenn man aber, nachdem er bereits verwelkt ist, die Zwiebel ausgräbt, so sieht man, da\& er an der Seite der Zwiebel entstanden ist, während die neue Zwiebel allmäblig heranwächst und den Saft der ganzen Pflanze an sich zieht. Diese Zeit ist die richtige zur Verpflanzung. ein Blatt.

Alle Tulpen, ehe sie blühen, sind stengellos und tragen nur

Wenn ein zweites eingerollt sich zeigt, ist Hoffnung, daß die Pflanze in diesem Jahr zur Blüte kommt.

Auch der starke Filzbelag der Innenseite der Zwiebelhülle ist Clusius nicht entgangen: er deutet ihn als weiches Lager der Zwiebel selbst.

Diese meisterhafte Arbeit schließt Clusius mit der Anekdote, daß vor einigen Jahren ein Antwerpener Kaufmann vielleicht über die im übrigen unbekannten medizinischen Eigenschaften der Tulpenzwiebeln hätte Auskunft geben können: Er erhielt solche von Konstantinopel mit einer Ladung Baumwolltuch; er hielt sie für Speisezwiebeln und ats einen Teil davon geröstet mit Essig und Ōl als Salat. Der Rest, den er wegwarf, ergab dann dem sie rettenden Herrn Georg Ry a von Mecheln die schönsten Tulpen. $O b$ und wie sich dies rege Interesse für Tulpen schlieflich zu dem großen Tulpenschwindel in Holland in den Dreißigerjahren des 17. Jahrhunderts auswuchs, darüber vergl. Solms-Laubach, a. a. 0 .

Narcissus persicus $=$ Sternbergia lutea erhielt Clusius aus Konstantinopel von Philipp de Bruxelles 1575, und noch immer taucht diese Herbstblüte hie und da in unseren älteren Gärten auf.

Auch einen großblütigen orientalischen Galanthus (S. 183) erhielt er von da, der im März 1583 blühte.

Ornithogalum arabicum kam aus gleicher Quelle zuerst nach Belgien, dann nach Wien, wo er 1580 zur Blüte kam und auch noch, aber selten, als Gartenplanze vorkommt. 
Hyacinthus comosus Byzantinus 195 blüte im gleichen Jahr und scheint eine weiße Bellevalia zu sein.

Rätselhaft bleibt auch der, schon in der spanischen Flora ausführlich behandelte und abgebildete, blau blühende Bulbus eriophorus, von dem Clusius bei seinem Freunde Aicholtz ein aus der Türkei durch Philibert de Bruxelles mitgebrachtes Exemplar in Blüte sah. Clusius behauptet, die Zwiebelschuppen seien mit so dichter Wolle bekleidet, daß sie zu Faden verarbeitet werden können?

Schon vor vielen Jahren ist durch Cortusus aus Padua nach Belgien als Tibcadi gesandt worden Muscari (M. moschatum), angeblich aus Gärten jenseits des Bosporus stammend. "Aus Konstantinopel kommt es als Muscari, Muschoromi oder Muscurimi", ein Name der auch in M us aeus Märchen wiederkehrt. Die herrlich duftende Pflanze ist wohl beute in den Gärten sehr selten geworden?

Gefüllte Tazetten 174 kamen schon 1575 nach Wien. Von den zwei aus dem Orient eingeführten Allium ist $210 \mathrm{~A}$. ophioscorodon, das nach Auger. de Bousbeque bei den Türken das beliebteste sei. 208 scheint einigermaßen mit $A$. ascalonicum zu stimmen. Es fällt auf, daß Clusius diese Laucharten als neue Erwerbungen anführt.

Crocus Moesiacus hat Stefan v. Hausen auf einer Rückreise von Konstantinopel in Serbien oberbalb Belgrad 1579 gefunden und eingeführt, eine heute noch allgemein gehegte Pflanze.

Iris susiana ist vor Weihnachten 1573 durch den Gesandten A ugerius de Bousbeque zum erstenmal nach Wien gebracht worden, aber die meisten Versuche, sie zu vervielfältigen, schlugen fehl, während sie in Belgien zur Blüte gebracht wurde.

Ranuncultus asiaticus kam dem Clusius 1580 durch den Herrn Chr. v. Entzesdorf zu, welcher eine Zeichnung nach Konstantinopel gesandt hatte, damit man dort wisse, welche Pflanze verlangt werde. Mehr konnte man wahrlich im Interesse des Hofbotanikers nicht tun. Aber Glusius hatte damit kein Glück; ein Hausdieb, der seinen Garten all seiner Zier beraubte, hat ihm auch den ersehnten Ranunkel bis auf zwei Pflänzlein geraubt, von denen nur eines zur Blüte kam. Bild 375 . Ein ungefülltes Exemplar ist als Tarabolos Catamer, Ranunkel von Tripolis, 376 abgebildet.

Eine ganze Monographie einfacher und gefüllter Gartenanemonen (A. coronaria, A. fulgens, $A$. stellata) von 9 Seiten und mit 4 Bildern, deren Einführungsgeschichte - sie stammen mit einer spanischen Ausnahme sämtlich aus der Türkei - mit diplomatischer Genanigkeit erðrtert ist und die schon in der spanischen Flora, S. 306, ibren Anfang nahm, wollen wir dem Spezialisten überlassen.

Mit heißem Bemühen und hoher Bewunderung setzt Clusius in der Pannon. Flora, S. 395, seine Studien über Admirabilis Peruanorum (Mirabilis Jalapa) fort, die er schon in seiner Schrift: Notae in Garciae aromat. Antwerp. 1582 begonnen hat. Die regellose Buntscheckigkeit der Blüten wird von ihm aufs genaneste beschrieben.

Er faßt die gescheckte Pflanze als den Typus und zwei Formen: II unicolor rubra und III Jasmin rosso, beide einfarbig rot, als Abänderungen auf und hält dafür, daß wenigstens II aus dem Samen des I'ypus erwachsen sei. Der Same sei aus Peru, wo die Pflanze Hachalindi, 
mit rauher Aspiration, genannt werde, nach Spanien gekommen. Bei den Mulierculae von Wien, die von ihr entzückt sind, heißt sie geschecket indianische Blumen und A. Cortusus in Padua gebraucht sie als eine, das Wasser trefflich ausführende Arznei. Vor 50 Jahren war die interessante Nyctaginee noch bei uns in Gärten zu sehen.

\section{Anhang: Aus dem Nachlaf des C. Clusius.}

Nach dem am 17. April 1609 in Leyden erfolgten Tode des Carl Clusius gab 1611 sein Antwerpener Freund und Verleger, der Buchdrucker Rapheleng, der Nachfolger des Christoph Plantin, eine Sammlung nachgelassener Notizen in einem Quartheft von 134 Seiten mit Holzschnitten heraus unter dem Titel: Caroli Clusii Atrebatis Curae posteriores. Sie enthalten gegen 100 Pflanzenarten, die teils neu beschrieben sind, teils unter Bezugnahme auf die bereits in den Werken des Clusius abgehandelten Arten Verbesserungen und Berichtigungen erfahren.

Besonders interessieren uns die vielen Korrespondenten, mit denen Clusius bis an sein Ende in Verkehr stand und die für den allgemeinen Eifer sprechen, den Clusius in ganz Europa für seine Studien zu wecken wußte.

Gewidmet ist die posthume Publikation dem Freunde des Clusius, Matthaeus Caccini, einem adeligen Florentiner, der schon vier Jahre lang die Botanik pflegte, einen auserlesenen Garten unterhielt und dem Clusius sehr viele Seltenheiten, namentlich Zwiebelpflanzen aus Konstantinopel und Abbildungen blühender Exemplare, so einer Hyacinthe mit beblättertem Schaft, p. 37, zusandte.

Johann Dortmannus, Apotheker aus Grönigen, sandte ihm Abbildungen dreier damals neuer Arten der dortigen Flora: Saxifraga hirculus (Icon., p. 11, nicht besonders gut, aber die Frucht ist richtig), Trifolium fragiferum (Icon., p. 73) und "Gladiolus palustris" (Icon., p. 74) oder nach heutiger T'erminologie Lobelia Dortmanna, deren hohle Blätter gut beschrieben sind.

Ferner genannt sind Joachim Venerius von Bordeaux, die Gebrüder Joh. Theod. und Joh. Israel de Bry in Frankfurt, Wilhelm Parduyn und Joh. Somer aus Middelburg in Walcheren, die ihm Iris aus Mauretanien brachten, der Arzt Dr. Augerius Clutius, der aus Libyen ebenfalls Iris mitnahm und aus Malaga das schöne Limonium Rauwolfianum (Icon., p. 63), unsere Statice sinuata. Nicolaus Fabricius de Peiresc, Mitglied des Parlaments von Aix, schickte eine Abbildung des Astragalus Massiliensis (Icon., p. 112) und Petrus Gassamas, Enkel des Christoph Plantin, der oberhalb Toulouse wohnte, eine Menge vielfarbiger, knolliger Iris aus den Pyrenäen. Besonders hervorgehoben wird der Kapuziner-Pater Gregorius de Reggio in Innsbruck, der 1608 dem Olusius ein ganzes Herbarium von Alpenpflanzen sandte, u. a. Primula glutinosa (Icon., p. 58) $=$ Auricula Ursi octava, reposita nimirum inter chartas cum aliis plantis alpinis e Tyrolensibus istis montibus erutis ab illo reverendo patre, dabei auch Pyramidalis Patris Gregorii da Reggio (Icon., p. 68, aber schlecht), welche wohl Campanula elatinoides von Brixen darstellt. Dieser Pater fand auch in Tirol ein Botrychium (Lunariae spec. seu Oplırys, p. 65), welches gar 
wohl das B. simplex Hitchk. sein könnte: Pater Gregorius istic obserrabat pumilum et tenellum quoddam plantae quam Ophrin vocant genus, cui sane ante simile conspicere non memini. Bekanntlich hat schon in den Stirp. Pannon., p. 511, Clusius das Botrychium Matricariae aus Schlesien als Lunaria minor ramosa gut abgebildet.

Besonders interessieren uns aber die zablreichen Rhizotomi, eigentliche Hausierer mit seltenen. lebenden Pflanzen für die Gärten besonders der Niederländer, ein Gewerbe, das damals blähte und uns den höchsten Begriff von dem Flor gibt, in welchem die Kultur schöner und rarer, besonders Zwiebelpflanzen stand. Unter vielen dieser Rhizotomi Galli es scheinen alle Franzosen gewesen zu sein - wird besonders genannt der Pariser Nicolaus de Quelt, der 1606 und 1608 Mengen von Iris, Scilla, Narcissus, Cyclamen, Eriophorum (= Scilla, besonders Peruviana), Fritillaria aus den Pyrenäen, Spanien, Portugal, Italien, Nordafrika sammelte und anbot, am meisten aber Gulielmus Boëlius, der aus Spanien z. B. die Viola arborescens mitbrachte. Namentlich entnahm Clusius den Körben (Sportulae) dieser Händler viel trockene Fragmente von Seltenheiten. Als Kunden dieser Vorläufer der heutigen Versandgeschäfte alpiner und orientalischer Pflanzen nennt Clusius den Christian Porretus, Apotheker in Leyden, die Frau v. Matenesse, den Jobn Hoghelandus, den Simon Touar und sich selbst. Einmal erschienen drei solcher Händler auf einmal.

Aus den Körben des Boëlius erhielt Clusius auch "Amourettes tremblantes", ein Gramen tremula panicula in zwei Arten, eine weißlich, die andere rauchgrau, vielleicht Eragrostis-Arten.

Aber auch die Niederländer Schiffer brachten reichlich Exemplare oder doch Albums mit, in denen sie Pflanzen nach dem Leben abgemalt hatten. So verdankte Clusius einem aus Brasilien zurückkehrenden Joh. ran Ufele treffliche Bilder des männlichen und des weiblichen Carica Papaya-Baumes (Mamoera mas et femina, p. 78 und 80) und diesmal irrt sich Clusius im Geschlechte nicht.

\section{Nekrologie des C. Clusius.}

Biographische Notizen über Clusius geben, und zwar recht eingehende, die zwei Schriften: 1. des Boisardus im II. Teil der Icones virorum illustrium, die aber nur bis 1593 reicht, 2. die sehr stilvolle akademische Oratio funebris des Professors der Medizin und Rektors der Akademie von Leyden, Everardus $\nabla$ orstius, die nach dem Begräbnis von Olusius in der Akademie am 7. April 1609 gehalten wurde.

Ich entnehme diesen beiden Dokumenten folgendes:

Die Eltern von Carl Clusius, geboren am 26. Februar 1526 in Arras, sind Michael de l'Escluse, Herr zu Watenes, Mitglied des Provinzialrates, und Wilhelmine Quineaut. Nach der ersten Schulzeit in der Vaterstadt kam der Knabe für zwei Jahre nach Gent und 1546 zum Studium der Rechte nach Löwen, wo er drei Jahre zubrachte und sich schon einen Namen machte. 22 Jahre alt, ging er auf Reisen nach Deutschland, hielt sich in Marburg auf, wo er den damals berühmtesten Juristen 0ldendorp hörte und mit dem Theologen Andr. Hyperius sich befreundete. Auf dessen Rat zog er nach einem Jahre nach Wittenberg, hauptsächlich um Philipp Melanchthon kennen zu lernen, 
der als das Orakel der Wissensehaft in deutschen Landen galt. Um das Jahr $15 \overline{5} 0$ zog er über Frankfurt, Straßburg, durch die Schweiz und die Waadt nach Lyon und Montpellier, wo er drei Jahre blieb und von dem berühmten Arzt Guilelmus Rondeletius als Wohn- und Tischgenosse aufgenommen wurde. Hier zuerst erfaßte ihn die Liebe zur Medizin und Pflanzenkunde, so daß er die Jurisprudenz aufgab. "Über alles Maß der Botanik ergeben", durchforschte er das ganze Narbonnesische Gallien, schenkte aber auch der Lage, den Altertümern, den Sitten des Volkes seine Aufmerksamkeit. Daselbst befreundete er sich „mit dem Fürsten der Dichter Deutschlands, Peter Lotiching, der ihm Verse widmete". Hier auch erlangte er den Grad des Lizenziaten in der Heilkunde. Wegen des Krieges zwischen Karl V. und Hein rich II. von Frankreich von seinem Vater zurückberufen, reiste er nun über Lyon nach Genf, wo er ein halbes Jahr "apud Allobrogos" verweilte, kam wiederum nach Basel, wo er von der Fakultät mit den ausgesuchtesten Ehren empfangen wurde, schiffte den Rhein hinab nach Köln und kam von da nach Antwerpen. Von 1555-1563 brachte er in den Niederlanden zu, außer daß er zwei Jahre in Paris sich auf hielt. Er übersetzte die von Donatus Acciaiolus lateinisch geschriebenen Leben des Hannibal und des Scipio ins Französische, auch die Geschichte der Pflanzen des Dodonaeus. Da die Umstände einer Reise nach Italien, wohin es ihn am meisten zog, nicht günstig waren, ging er nach Augsburg und von da im Geleite der Gebrüder Fugger durch Belgien und das westliche Frankreich nach Spanien, das er gründlich bereiste und während eines Jahres von den Pyrenäen bis an die Meerenge von Cadiz erforschte. In Lissabon fielen ihm von ungefähr die portugiesisch geschriebenen Dialoge des Garçias ab Orto über die Gewürze Indiens in die Hände, die er ins Latein übertrug, mit Noten versah und 1564 bei $\mathrm{Ch}$. Plantin in Antwerpen herausgab; er fügte auch die Übersetzung der Heilmittel der Neuen Welt aus dem Spanischen des Sevillaner Arztes Nikolaus Monardes bei. 1565 war er in Belgien zurück und blieb da, umgeben von trefflichen Freunden: Boysott, Brancion, Gebr. Laurin, Plantin, Rapheleng u. a. bis 1570. $1571 \mathrm{zog}$ er über Paris und Calais nach England. Nach seiner Rückkehr 1573 erging an ihn der ehrenvolle Ruf des Kaisers Max II. nach Wien als kaiserlicher Hofrat und Direktor des kaiserlichen Gartens, welches Amt er 14 Jahre lang sowohl unter Max als dessen Sohn Rudolf II. bekleidete. Unablässig bereiste er von Wien aus die Gebirge Österreichs und Ungarns und gab hier sowohl seine Stirp. Hispan. 1576 als die Stirp. Pannon. 1583 heraus. Am gleichen Hofe lebten seine Freunde Joh. Sambucus, Jul. Alexandrinus, Crato Dodonaeus, auch war er intim mit den ungarischen Magnaten Graf Serotin und Baron Batthyan. Von Wien aus machte er eine zweite Reise nach England, we er den Umgang mit Philipp Sidney und Francis Drake genok, der sowie seine Schiffsgenossen ihm viele exotische Seltenheiten verehrten.

Des Hoflebens müde, siedelte Clusius 1587 nach Frankfurt a. M. über, wo er sechs Jahre zurückgezogen lebte, oft zu Besuch bei dem Landgrafen $W$ ilhelm von Hessen, der ihn durch ein Jahresgehalt ehrte. Hier übersetzte er aus dem Französischen die Observationes des 
P. Bellonius über den Orient und gab sie 1589 heraus. 1603 wurde er, schon 77 Jahre alt, von den Generalstaaten an die Akademie nach Leyden berufen, wo er täglich Vorlesungen hielt und alle seine Werke in zwei Foliobänden zusammenzog: Histor. rariorum Plantarum und Exot. Clusius starb am 4. April 1609, 84 Jahre alt, unverheiratet, hoch geschätzt wegen seiner Charaktereigenschaften. Er war von phänomenalem Fleiß, fromm, wohltätig; er überließ - ein seltenes Beispiel - seinem jüngeren Bruder sein Majorat Watenes; er war von unglaublicher Ruhe und Heiterkeit des Geistes: unam eandemque perpetuo premente viam. Gegen Ende seines Lebens waren Jos. Sealiger und Vincentius Pinellus seine nächsten Freunde. Und doch war bei einem so tätigen Leben seine Gesundheit von Jugend an schwankend und nicht blühend und die Biographen zählen genau alle seine zahlrejchen Unfälle auf, die ihm das Reisen beschwerlich machten: Ganz jung litt er mehrere Jahre lang beständig an Fiebern, 24 Jahre alt hatte er Wassersucht, von der ihn Rondelet kurierte. Bei Gibraltar fiel er vom Pferde und brach den rechten Arm über dem Ellenbogen sowie auch das rechte Bein. In Wien erlitt er, 55 Jahre alt, die Luxation des linken Beines und einen Knöchelbruch. In Frankfurt verrenkte er das rechte Hüftgelenk und blieb lahm, so daß er sich zweier Krücken bedienen mußte und vom vielen unbeweglichen Sitzen and Liegen sich Stein- und Bruchleiden einstellten. Aber sein Gesicht blieb, gleich seinem Geist, bis zum Tode scharf. Nicht weniger als 16 dichterische Nachrufe sind der Leichenrede des Rektors beigedruckt, wovon zwei in griechischen, die anderen in lateinischen Versen.

\title{
Verzeichnis der von mir gemachten Angaben zur Systematik und Synonymio der Pilze.
}

\author{
Von Prof. Dr. Franz von Höhnel (Wien).
}

\section{Vorbemerkungen.}

Im Laufe meiner nunmehr zwölijäbrigen Tätigkeit auf dem Gebiete der speziellen Mykologie babe ich eine große Menge von systematischen und synonymischen Tatsachen festgestellt, die zum Teile noch der Veröffentlichung harren, zum Teile in meinen 53 Publikationen enthalten sind. Da letztere an neun verschiedenen Orten zur Veröffentlichung kamen, ist die Auffindung der gemachten Angaben selbst für den Kenner meiner Arbeiten mit Schwierigkeiten und Umständlichkeiten verbunden, weshalb eine Zusammenstellung der wesentlichsten derselben nicht nur von Nutzen sein wird, sondern geradezu zu einer Notwendigkeit geworden ist. Dies ist umsomehr der Fall, als der größere Teil derselben in den bisher erschienenen Bänden von Sa ceardo's Sylloge Fungorum nicht aufgenommen wurde und auch in den weiter erscheinenden Bänden dieses Sammelwerkes nicht erscheinen wird.

Daher ist diese Publikation eine notwendige Ergänzung der Sylloge Fungorum.

Die nachfolgende Zusammenstellung enthält (I.) ein vollständiges Verzeichnis meiner Arbeiten mit Angabe des Publikationsortes, dann (II.) 